\title{
Detection of Aspergillus DNA by a nested PCR assay is able to improve the diagnosis of invasive aspergillosis in paediatric patients
}

\author{
Correspondence \\ Margit Hummel \\ Margithummel@yahoo.de
}

Received 20 February 2009

Accepted 12 June 2009

\author{
Margit Hummel, ${ }^{1}$ Birgit Spiess, ${ }^{1}$ Julia Roder, ${ }^{1}$ Gregor von Komorowski, ${ }^{2}$ \\ Matthias Dürken, ${ }^{2}$ Karim Kentouche, ${ }^{3}$ Hans J. Laws, ${ }^{4}$ Handan Mörz, ${ }^{1}$ \\ Ruediger Hehlmann ${ }^{1}$ and Dieter Buchheidt ${ }^{1}$
${ }^{1}$ III. Medizinische Universitätsklinik, Medizinische Fakultät Mannheim, Universität Heidelberg, D-68167 Mannheim, Germany
${ }^{2}$ Klinik für Kinderheilkunde, Klinikum Mannheim, Universität Heidelberg, D-68167 Mannheim, Germany
${ }^{3}$ Klinik für Kinder- und Jugendmedizin, Friedrich-Schiller-Universität Jena, D-07743 Jena, Germany
${ }^{4}$ Klinik für Kinderonkologie, -hämatologie und Klinische Immunologie, Düsseldorf, Germany

\begin{abstract}
Fungal infections are a leading cause of morbidity and mortality in severely immunocompromised patients and have been increasing in incidence in recent years. Invasive aspergillosis (IA) is the most common filamentous fungal infection and is, in adults as well as in children, difficult to diagnose. Several PCR assays to detect Aspergillus DNA have been established, but so far, studies on molecular tools for the diagnosis of IA in children are few. We evaluated the results of a nested PCR assay to detect Aspergillus DNA in clinical samples from paediatric and adolescent patients with suspected IA. Blood and non-blood samples from immunocompromised paediatric and adolescent patients with suspected invasive fungal infection were sent for processing Aspergillus PCR to our laboratory. PCR results from consecutive patients from three university children's hospitals investigated between November 2000 and January 2007 were evaluated. Fungal infections were classified according to the EORTC classification on the grounds of clinical findings, microbiology and radio-imaging results. Two hundred and ninety-one samples from 71 patients were investigated for the presence of Aspergillus DNA by our previously described nested PCR assay. Two, 3 and 34 patients had proven, probable and possible IA, respectively. Sensitivity (calculated from proven and probable patients, $n=5$ ) and specificity (calculated from patients without IA, $n=32$ ) rates of the PCR assay were 80 and $81 \%$, respectively. Our nested PCR assay was able to detect Aspergillus DNA in blood, cerebrospinal fluid and bronchoalveolar lavage samples from paediatric and adolescent patients with IA with high sensitivity and specificity rates.
\end{abstract}

\section{INTRODUCTION}

Invasive fungal infections (IFIs) are a major clinical problem and a leading cause of morbidity and mortality in severely immunocompromised patients, especially in patients with haematological malignancies and intensive antineoplastic treatment.

Invasive aspergillosis (IA) is the most common filamentous fungal infection observed in these patients (El Mahallawy et al., 2002; Hovi et al., 2000). The incidence of IA has increased significantly in recent years in parallel

Abbreviations: BAL, bronchoalveolar lavage; CNS, central nervous system; CSF, cerebrospinal fluid; GM, galactomannan; IA, invasive aspergillosis; IFI, invasive fungal infection. with the increasing number and improved survival of immunocompromised patients (Denning, 1998; Marr et al., 2002). While IA in adults has been well characterized, there has been little investigation into IA in paediatric patients (Roilides, 2006; Steinbach, 2005a, b). In a large retrospective cohort study including 152231 immunocompromised children in the United States, the annual incidence of IA was found to be $0.4 \%$. The highest incidence of IA was seen in children who had undergone allogeneic bone marrow transplantation $(4.5 \%)$ and those with acute myelogenous leukaemia (4\%) (Zaoutis et al., 2006). Other predisposing host factors are acquired immunodeficiency syndromes, chronic granulomatous disease, severe combined immunodeficiency, organ transplantation, granulocytopenia, corticosteroid and other 
immunosuppressive therapies and prematurity (Walsh et al., 1996).

Morbidity and mortality of IFI are still high even though new antifungal drugs have brought some improvement (Herbrecht et al., 2002a; Upton \& Marr, 2006; Walsh et al., 2004b). A recent Italian multicentre surveillance study including paediatric cancer patients reported a mortality rate of $34 \%$ in children with deep tissue infection (Castagnola et al., 2006). To improve the outcome for patients with IA, early diagnosis and treatment initiation is important but establishing a reliable diagnosis remains difficult. In the majority of cases, the diagnosis of IA is made at autopsy (Chamilos et al., 2006; Groll et al., 1996; Lin et al., 2001; Subira et al., 2003). Immunocompromised children rarely display typical inflammatory responses. Radio-imaging is not specific and is difficult to interpret especially at the early stage of IA. Invasive diagnostic procedures can often not be performed because of the severity of the underlying disease and the risk of bleeding complications in thrombocytopenic patients. Numerous PCR assays to detect fungal DNA have been described, but most studies focus on adult patient cohorts (Baskova et al., 2007; Florent et al., 2006; Halliday et al., 2006; Hummel \& Buchheidt, 2007; Jordanides et al., 2005; SchabereiterGurtner et al., 2007; White et al., 2006). Studies on the molecular diagnosis of IFIs in children are few (Roilides, 2006). Some studies have included children in larger cohorts, but results were not stratified for paediatric patients (Buchheidt et al., 2001; Maertens et al., 2001; Pinel et al., 2003). From the data available, both galactomannan (GM) ELISA and PCR protocols appear to be less useful in children than in adults (Bochennek et al., 2006).

To assess the role of molecular diagnosis of IA in children, we evaluated the results of a nested PCR to detect Aspergillus DNA in samples from paediatric and adolescent patients with IA.

\section{METHODS}

Patients and samples. Clinical samples from paediatric and adolescent patients with suspected IFI were sent for processing Aspergillus PCR to our laboratory. PCR results from all consecutive patients from three university children's hospitals investigated between November 2000 and January 2007 were evaluated in this study. The majority of patients had malignant haematological diseases. Neutropenia (absolute neutrophil count $<500 \mu^{-1}$ ) was present in $38(54 \%)$ of the patients. Patients' characteristics are shown in Table 1. The age of the youngest patient was 1 month. There were only three patients aged above 18 years included in the study. All of these patients were treated in a paediatric hospital according to paediatric chemotherapy protocols and were therefore included in the study. All samples were obtained during routine diagnostic procedures under sterile conditions and after informed consent of the patients or the parents. Samples were shipped to our laboratory in sterile tubes and processed immediately. Fungal infections were classified according to the EORTC classification (Ascioglu et al., 2002) on the grounds of symptoms and clinical findings, microbiology and radio-imaging results.
Table 1. Patients' characteristics

\begin{tabular}{|lc|}
\hline Age, median & 8 years \\
& (range $0-20)$ \\
Female/male & $27 / 44$ \\
Underlying immunocompromising condition & $n=71$ \\
Acute lymphoblastic leukaemia & 22 \\
Acute myeloid leukaemia & 5 \\
Allogeneic haematological stem cell & 17 \\
transplantation* & \\
Burkitt's lymphoma & 3 \\
Cystic fibrosis & 4 \\
Solid tumours & 12 \\
Congenital immunodeficiency & 1 \\
Chronic granulomatous disease & 2 \\
Others $\dagger$ & 5 \\
\end{tabular}

*Allogeneic haematological stem cell transplantation was performed for acute leukaemia $(n=9)$, severe combined immunodeficiency $(n=2)$, thalassaemia $(n=2)$, chronic granulomatous disease $(n=1)$, Hodgkin's lymphoma $(n=1)$, secondary chronic myelomonocytic leukaemia $(n=1)$ and Ewing's sarcoma $(n=1)$.

†Others include Langerhans cell histiocytosis, juvenile chronic arthritis, haemophagocytic lymphohistiocytosis, respiratory distress syndrome and prolonged intensive care treatment.

PCR. Aspergillus DNA was detected in clinical samples by an experimentally and clinically validated nested PCR assay as described previously (Buchheidt et al., 2001, 2002; Hummel et al., 2004; Skladny et al., 1999). To minimize the risk of contamination, all samples were handled under sterile conditions in a laminar flow cabinet. Between $0.5 \mathrm{ml}$ [cerebrospinal fluid (CSF)] and $5 \mathrm{ml}$ [blood, bronchoalveolar lavage (BAL)] was used for the PCR assay. For the nested two-step PCR technique, two pairs of oligonucleotide primers (AFU 7S and AFU 7AS for the first step and AFU 5 S and AFU 5AS for the second step) derived from sequences of the Aspergillus fumigatus 18S rRNA gene (GenBank accession no. AB008401) and specific for Aspergillus species were used. The detection limit of the nested PCR assay is $1-5$ c.f.u. ( $\mathrm{ml} \mathrm{blood})^{-1}$.

The definition of IA was based on the 2002 EORTC/MSG guidelines (Ascioglu et al., 2002). Decisions about whether patients had proven, probable or possible IA were made strictly independently from PCR results. The PCR results were evaluated by correlating them with the clinical classification. Results of serological diagnostic techniques (GM assay, Platelia Aspergillus EIA; Bio-Rad) and post-mortem histological examination were included for clinical classifications.

Statistical analysis. Continuous variables such as age were summarized using descriptive summary statistics ( $n$, median, mean, range). Categorical variables such as PCR results were tabulated and presented as a percentage. For calculation of sensitivity of the PCR assay, episodes with proven and probable IA were assumed to be true-positive episodes. Episodes with possible IA are non-conclusive and were therefore not considered in the calculation of sensitivity and specificity of the PCR assay. For calculation of specificity, episodes without evidence of IA were assumed to be true-negative episodes. Statistical analysis was performed with sPSS Advanced Statistics, version 10.0.6.

\section{RESULTS AND DISCUSSION}

Two hundred and ninety-one blood and non-blood samples from 71 patients were sent to our laboratory for 
Table 2. Origin of samples, sample volumes and PCR results

\begin{tabular}{|c|c|c|c|c|c|}
\hline CSF & $1(0.5-2)$ & 43 & 11 & 29 & 3 \\
\hline BAL & $3(2-5)$ & 14 & 2 & 12 & 0 \\
\hline Liver biopsy & & 4 & 0 & 4 & 0 \\
\hline Bone marrow aspirate & $3(3-4)$ & 3 & 0 & 3 & 0 \\
\hline Sinus aspirate & $0.65(0.5-0.8)$ & 2 & 0 & 2 & 0 \\
\hline Skin swab & & 1 & 0 & 1 & 0 \\
\hline
\end{tabular}

PCR from three paediatric centres. Two hundred and eighty-five samples were evaluable. The median number of samples obtained per patient was two (range 1-41). Thirtyfour samples gave positive PCR results and 251 samples gave negative PCR results.

Aspergillus DNA was detected in blood, CSF and BAL samples. Table 2 shows the source of the samples and PCR results.

\section{Proven and probable IA, and sensitivity of the PCR assay}

In our cohort of paediatric and adolescent patients, IA could be classified in only five patients as 'proven' $(n=2)$ or 'probable' $(n=3)$, reflecting the difficulties of verifying the diagnosis of IA (Table 3). All patients with proven or probable IA had acute leukaemia; one had undergone allogeneic haematological stem cell transplantation for acute myeloid leukaemia. Three patients had disseminated IA; in two of these there was involvement of the central nervous system (CNS). Table 4 shows the characteristics of patients with proven and probable IA. In patients with proven and probable IA, Aspergillus DNA was detected by PCR in blood, CSF and BAL samples. Four of five patients with probable or proven IA had at least one positive PCR result. Assuming that the patients with proven and probable IA were 'true positives', the sensitivity of PCR testing was $80 \%$. It is difficult to calculate sensitivity and specificity rates with small numbers of patients, but most studies report only few patients with proven or probable
IA, even if data are collected over a long time interval (Hovi et al., 2007).

The patient with proven IA but negative PCR results had IA affecting the liver and the kidney. IA was proven from a liver biopsy specimen, which was positive for A. fumigatus. Only sputum and blood samples were investigated by PCR in this patient. An explanation for the PCR negativity is that the samples were not obtained from the site of infection and that there was no DNAaemia at the time of blood sampling, so that Aspergillus DNA was not present in blood samples or the level of DNAaemia was below the detection threshold of the PCR assay. There is still relatively poor understanding of DNA release and kinetics in fungal infections (Hope et al., 2005).

In three cases with proven or probable disease, samples were obtained from the site of the fungal infection. In two patients with cerebral aspergillosis, CSF samples contained Aspergillus DNA and in another patient with pulmonary aspergillosis, a BAL sample was positive for Aspergillus DNA. This supports the hypothesis that investigating samples from the site of Aspergillus infection is more sensitive than blood sampling (Hummel \& Buchheidt, 2007).

The majority of samples obtained from patients with proven, probable and possible IA were obtained during antifungal treatment. Mennink-Kersten et al. (2006) detected in their in vitro studies fungal DNA during autolysis caused by nutrient limitation or antifungal treatment. However, not all PCR samples were positive

Table 3. PCR results according to EORTC/MSG groups

\begin{tabular}{|lcccr|}
\hline & Proven & Probable & Possible & No IA \\
\hline No. of patients & 2 & 3 & 34 & 32 \\
No. of patients with positive PCR results & 1 & 3 & 11 & 6 \\
No. of patients with negative PCR results & 1 & 0 & 22 & 26 \\
\hline
\end{tabular}

${ }^{\star}$ In the group of patients with possible IA, the samples of one patient were not evaluable. 


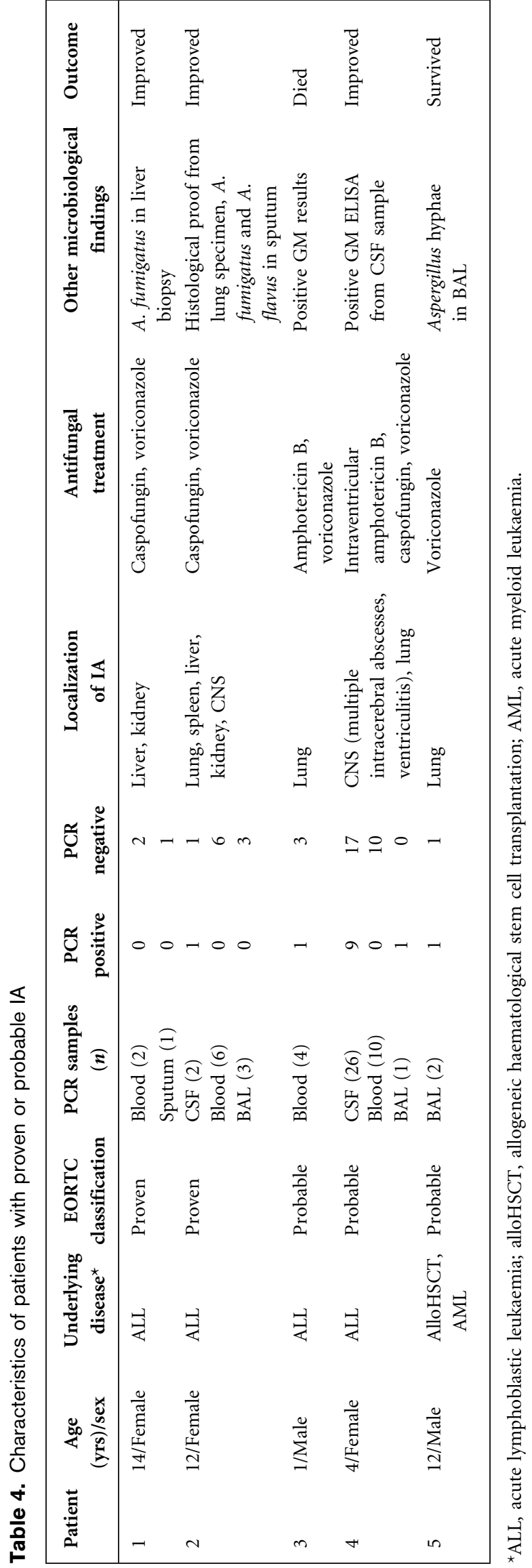

during the lytical phase. The detection of fungal DNA is certainly different in an in vitro setting as reported by Mennink-Kersten et al. (2006) as compared to the clinical setting where there is still relatively poor understanding of DNA release and kinetics. Several clinical studies report that the sensitivity of PCR testing for Aspergillus infections is limited during antifungal treatment due to a decrease of fungaemia (Kami et al., 2001; Lass-Florl et al., 2004; O'Sullivan et al., 2003). This may account for false-negative PCR results. This observation is supported by the course of PCR results in a patient of our study cohort where serial sampling for PCR testing was possible. In this patient (patient 4, Table 4) with disseminated IA with involvement of the CNS and the lungs, an Ommaya reservoir was implanted because of increased intracranial pressure. Serial CSF samples $(n=26)$ were investigated by PCR for Aspergillus DNA. Initially, 8/9 serial CSF samples were positive for Aspergillus DNA. Samples became negative during antifungal treatment with intraventricular amphotericin, voriconazole and caspofungin, accompanied by clinical improvement.

So far, most studies on Aspergillus PCR testing have included only adult patients with only a few studies reporting on paediatric patients. Challier et al. (2004) report a sensitivity of an $A$. fumigatus-specific real-time PCR assay of $100 \%$ and $50 \%$ in children with proven and probable IA, respectively. In the study of El-Mahallawy et al. (2006) using a pan-fungal PCR assay in paediatric high-risk cancer patients for the diagnosis of yeast and mould infections, the sensitivity of PCR testing was $75 \%$.

\section{Patients with possible IA}

In 34 patients, IA was classified as 'possible'. Among patients with possible IA, 11/34 had at least one positive PCR result. Of these, four had CNS lesions and three had disseminated disease. In one patient with possible IA of the CNS, Aspergillus DNA was detected in a CSF sample, but other microbiological investigations were negative.

It remains unclear whether some of these patients actually had IA that could not be proven, as especially CNS aspergillosis is difficult to diagnose (Cunha, 2001; McGinnis, 1983). The EORTC/MSG definition of 'possible IFI' is the least specific: in this disease category it is uncertain whether IFI is present. In a series of 21 paediatric patients who underwent early surgical lung resection in pulmonary or disseminated IA, 12 patients were classified as having possible IA before surgery. Thereof, in 10 patients IFI was confirmed at surgery (Cesaro et al., 2007). Subira et al. (2003) reported that $60 \%$ of IA cases proven at autopsy were classified as 'possible IA' before patients' death.

Due to the uncertain disease status in the group of 'possible IFI', the statistical performance of PCR testing was calculated using different definitions of disease status (Table 5). 
Table 5. Performance of Aspergillus PCR with use of different definitions of disease status

Data are percentage $(95 \% \mathrm{CI})$. Under definition A, proven/probable cases are true positives, at-risk cases are true negatives and possible cases are excluded from statistical analysis; under definition B, proven/probable/possible cases are true positives and at-risk cases are true negatives; under definition $\mathrm{C}$, proven/probable cases are true positives and possible/at-risk cases are true negatives.

\begin{tabular}{|lccl|}
\hline \multirow{2}{*}{ Value } & \multicolumn{4}{c|}{ Invasive aspergillosis definition } \\
\cline { 2 - 4 } & \multicolumn{1}{c}{ A } & B & C \\
\hline Sensitivity & $80(38-96)$ & $38(25-54)$ & $80(38-96)$ \\
Specificity & $81(65-91)$ & $81(65-91)$ & $73(61-82)$ \\
Positive predictive value & $40(17-69)$ & $71(50-86)$ & $19(8-40)$ \\
Negative predictive value & $96(82-99)$ & $53(39-66)$ & $98(89-100)$ \\
\hline
\end{tabular}

\section{Specificity of the PCR assay}

The initially considered differential diagnosis of IA could not be confirmed in 32 patients in the course of the disease and they were therefore classified as having no IA. Among patients without IA, 26/32 had negative PCR results, resulting in a specificity of $81 \%$. False-positive PCR results can be due to contamination. Aspergillus is a ubiquitously spread organism and therefore contamination is a considerable risk in spite of appropriate precautions in handling samples.

The specificity of PCR testing in our study was distinctly higher than the specificity of GM reported in other studies (Herbrecht et al., 2002b; Mennink-Kersten et al., 2004; Siemann \& Koch-Dorfler, 2001). The problem with GM ELISA in children is the high rate of false-positive results. Specificity rates of as low as $47.6 \%$ have been reported (Herbrecht et al., 2002b). This has been attributed to the predominantly milk-based diet of children, as GM is present in milk and may translocate across the intestinal wall if chemotherapy-induced mucositis is present. Also, lipoglycan from Bifidobacterium bifidum as well as drugs of fungal origin such as piperacillin-tazobactam may cause false-positive GM results (Steinbach et al., 2007; Sulahian et al., 2003; Viscoli et al., 2004; Walsh et al., 2004a). In a prospective study on Aspergillus GM antigen testing in paediatric haematopoietic stem cell transplant recipients, Steinbach et al. (2007) reported a specificity for GM testing of $98.4 \%$ after excluding samples from patients who were receiving piperacillin-tazobactam. With better specificity rates, Aspergillus PCR as a diagnostic test in invasive fungal disease is complementary to GM testing and other diagnostic tests.

\section{Conclusions}

PCR for Aspergillus DNA is a useful tool in establishing the diagnosis of IA with high sensitivity and specificity rates. Our nested PCR assay is able to detect Aspergillus DNA in blood, CSF and BAL samples. However, PCR detection in blood samples is much less sensitive. Small sample volumes as low as $0.5 \mathrm{ml}$ were sufficient for DNA detection. This is of advantage in small children where it is difficult to obtain appropriate specimens due to their small size (Roilides, 2006).

To increase the sensitivity of both PCR and serology testing, obtaining samples before the institution of antifungal treatment and serial sampling are desirable.

The combined use of culture, GM ELISA and PCR should result in an earlier and more definite diagnosis of IA in children. The diagnosis of IA remains a combination of the clinical presentation, findings on radio-imaging and results of conventional microbiological and molecular methods. PCR for Aspergillus DNA makes an essential contribution to the improvement of the diagnosis of IA.

\section{REFERENCES}

Ascioglu, S., Rex, J. H., de Pauw, B., Bennett, J. E., Bille, J., Crokaert, F., Denning, D. W., Donnelly, J. P., Edwards, J. E. \& other authors (2002). Defining opportunistic invasive fungal infections in immunocompromised patients with cancer and hematopoietic stem cell transplants: an international consensus. Clin Infect Dis 34, 7-14.

Baskova, L., Landlinger, C., Preuner, S. \& Lion, T. (2007). The PanAC assay: a single-reaction real-time PCR test for quantitative detection of a broad range of Aspergillus and Candida species. J Med Microbiol 56, 1167-1173.

Bochennek, K., Abolmaali, N., Wittekindt, B., Schwabe, D., Klingebiel, T. \& Lehrnbecher, T. (2006). Diagnostic approaches for immunocompromised paediatric patients with pulmonary infiltrates. Clin Microbiol Infect 12, 199-201.

Buchheidt, D., Baust, C., Skladny, H., Ritter, J., Suedhoff, T., Baldus, M., Seifarth, W., Leib-Moesch, C. \& Hehlmann, R. (2001). Detection of Aspergillus species in blood and bronchoalveolar lavage samples from immunocompromised patients by means of 2-step polymerase chain reaction: clinical results. Clin Infect Dis 33, 428-435.

Buchheidt, D., Baust, C., Skladny, H., Baldus, M., Brauninger, S. \& Hehlmann, R. (2002). Clinical evaluation of a polymerase chain reaction assay to detect Aspergillus species in bronchoalveolar lavage samples of neutropenic patients. $B r J$ Haematol 116, 803-811.

Castagnola, E., Cesaro, S., Giacchino, M., Livadiotti, S., Tucci, F., Zanazzo, G., Caselli, D., Caviglia, I., Parodi, S. \& other authors (2006). Fungal infections in children with cancer: a prospective, multicenter surveillance study. Pediatr Infect Dis J 25, 634-639.

Cesaro, S., Cecchetto, G., de Corti, F., Dodero, P., Giacchino, M., Caviglia, I., Fagioli, F., Livadiotti, S., Salin, F. \& other authors (2007). Results of a multicenter retrospective study of a combined medical and surgical approach to pulmonary aspergillosis in pediatric neutropenic patients. Pediatr Blood Cancer 49, 909-913.

Challier, S., Boyer, S., Abachin, E. \& Berche, P. (2004). Development of a serum-based Taqman real-time PCR assay for diagnosis of invasive aspergillosis. J Clin Microbiol 42, 844-846.

Chamilos, G., Luna, M., Lewis, R. E., Bodey, G. P., Chemaly, R., Tarrand, J. J., Safdar, A., Raad, I. I. \& Kontoyiannis, D. P. (2006). Invasive fungal infections in patients with hematologic malignancies in a tertiary care cancer center: an autopsy study over a 15 -year period (1989-2003). Haematologica 91, 986-989. 
Cunha, B. A. (2001). Central nervous system infections in the compromised host: a diagnostic approach. Infect Dis Clin North Am 15, 567-590.

Denning, D. W. (1998). Invasive aspergillosis. Clin Infect Dis 26, 781803.

El Mahallawy, H. A., Attia, I., Ali-el-Din, N. H., Salem, A. E. \& Abo-elNaga, S. (2002). A prospective study on fungal infection in children with cancer. J Med Microbiol 51, 601-605.

El Mahallawy, H. A., Shaker, H. H., Ali, H. H., Mostafa, T. \& Razak Abo-Sedah, A. (2006). Evaluation of pan-fungal PCR assay and Aspergillus antigen detection in the diagnosis of invasive fungal infections in high risk paediatric cancer patients. Med Mycol 44, 733739.

Florent, M., Katsahian, S., Vekhoff, A., Levy, V., Rio, B., Marie, J. P., Bouvet, A. \& Cornet, M. (2006). Prospective evaluation of a polymerase chain reaction-ELISA targeted to Aspergillus fumigatus and Aspergillus flavus for the early diagnosis of invasive aspergillosis in patients with hematological malignancies. J Infect Dis 193, 741-747.

Groll, A. H., Shah, P. M., Mentzel, C., Schneider, M., Just-Nuebling, G. \& Huebner, K. (1996). Trends in the postmortem epidemiology of invasive fungal infections at a university hospital. J Infect 33, 23-32.

Halliday, C., Hoile, R., Sorrell, T., James, G., Yadav, S., Shaw, P., Bleakley, M., Bradstock, K. \& Chen, S. (2006). Role of prospective screening of blood for invasive aspergillosis by polymerase chain reaction in febrile neutropenic recipients of haematopoietic stem cell transplants and patients with acute leukaemia. Br J Haematol 132, 478-486.

Herbrecht, R., Denning, D. W., Patterson, T. F., Bennett, J. E., Greene, R. E., Oestmann, J. W., Kern, W. V., Marr, K. A., Ribaud, P. \& other authors (2002a). Voriconazole versus amphotericin B for primary therapy of invasive aspergillosis. N Engl J Med 347, 408-415.

Herbrecht, R., Letscher-Bru, V., Oprea, C., Lioure, B., Waller, J., Campos, F., Villard, O., Liu, K. L., Natarajan-Ame, S. \& other authors (2002b). Aspergillus galactomannan detection in the diagnosis of invasive aspergillosis in cancer patients. J Clin Oncol 20, 1898-1906.

Hope, W. W., Walsh, T. J. \& Denning, D. W. (2005). Laboratory diagnosis of invasive aspergillosis. Lancet Infect Dis 5, 609-622.

Hovi, L., Saarinen-Pihkala, U. M., Vettenranta, K. \& Saxen, H. (2000). Invasive fungal infections in pediatric bone marrow transplant recipients: single center experience of 10 years. Bone Marrow Transplant 26, 999-1004.

Hovi, L., Saxen, H., Saarinen-Pihkala, U. M., Vettenranta, K., Meri, T. \& Richardson, M. (2007). Prevention and monitoring of invasive fungal infections in pediatric patients with cancer and hematologic disorders. Pediatr Blood Cancer 48, 28-34.

Hummel, M. \& Buchheidt, D. (2007). Molecular and serological diagnosis of invasive aspergillosis: new answers to old questions? Mycoses 50 (Suppl. 1), 18-23.

Hummel, M., Baust, C., Kretschmar, M., Nichterlein, T., Schleiermacher, D., Spiess, B., Skladny, H., Morz, H., Hehlmann, R. \& Buchheidt, D. (2004). Detection of Aspergillus DNA by a nested PCR assay is superior to blood culture in an experimental murine model of invasive aspergillosis. J Med Microbiol 53, 803-806.

Jordanides, N. E., Allan, E. K., McLintock, L. A., Copland, M., Devaney, M., Stewart, K., Parker, A. N., Johnson, P. R., Holyoake, T. L. \& Jones, B. L. (2005). A prospective study of real-time panfungal PCR for the early diagnosis of invasive fungal infection in haematooncology patients. Bone Marrow Transplant 35, 389-395.

Kami, M., Fukui, T., Ogawa, S., Kazuyama, Y., Machida, U., Tanaka, Y., Kanda, Y., Kashima, T., Yamazaki, Y. \& other authors (2001). Use of real-time PCR on blood samples for diagnosis of invasive aspergillosis. Clin Infect Dis 33, 1504-1512.
Lass-Florl, C., Gunsilius, E., Gastl, G., Bonatti, H., Freund, M. C., Gschwendtner, A., Kropshofer, G., Dierich, M. P. \& Petzer, A. (2004). Diagnosing invasive aspergillosis during antifungal therapy by PCR analysis of blood samples. J Clin Microbiol 42, 4154-4157.

Lin, S. J., Schranz, J. \& Teutsch, S. M. (2001). Aspergillosis casefatality rate: systematic review of the literature. Clin Infect Dis 32, 358-366.

Maertens, J., Verhaegen, J., Lagrou, K., Van Eldere, J. \& Boogaerts, M. (2001). Screening for circulating galactomannan as a noninvasive diagnostic tool for invasive aspergillosis in prolonged neutropenic patients and stem cell transplantation recipients: a prospective validation. Blood 97, 1604-1610.

Marr, K. A., Carter, R. A., Crippa, F., Wald, A. \& Corey, L. (2002). Epidemiology and outcome of mould infections in hematopoietic stem cell transplant recipients. Clin Infect Dis 34, 909-917.

McGinnis, M. R. (1983). Detection of fungi in cerebrospinal fluid. Am J Med 75, 129-138.

Mennink-Kersten, M. A., Klont, R. R., Warris, A., Op den Camp, H. J. \& Verweij, P. E. (2004). Bifidobacterium lipoteichoic acid and false ELISA reactivity in aspergillus antigen detection. Lancet 363, 325-327.

Mennink-Kersten, M. A., Ruegebrink, D., Wasei, N., Melchers, W. J. \& Verweij, P. E. (2006). In vitro release by Aspergillus fumigatus of galactofuranose antigens, 1,3- $\beta$-D-glucan, and DNA, surrogate markers used for diagnosis of invasive aspergillosis. J Clin Microbiol 44, 1711-1718.

O'Sullivan, C. E., Kasai, M., Francesconi, A., Petraitis, V., Petraitiene, R., Kelaher, A. M., Sarafandi, A. A. \& Walsh, T. J. (2003). Development and validation of a quantitative real-time PCR assay using fluorescence resonance energy transfer technology for detection of Aspergillus fumigatus in experimental invasive pulmonary aspergillosis. J Clin Microbiol 41, 5676-5682.

Pinel, C., Fricker-Hidalgo, H., Lebeau, B., Garban, F., Hamidfar, R., Ambroise-Thomas, P. \& Grillot, R. (2003). Detection of circulating Aspergillus fumigatus galactomannan: value and limits of the Platelia test for diagnosing invasive aspergillosis. J Clin Microbiol 41, 2184-2186.

Roilides, E. (2006). Early diagnosis of invasive aspergillosis in infants and children. Med Mycol 44 (Suppl. 1), 199-205.

Schabereiter-Gurtner, C., Selitsch, B., Rotter, M. L., Hirschl, A. M. \& Willinger, B. (2007). Development of novel real-time PCR assays for detection and differentiation of eleven medically important Aspergillus and Candida species in clinical specimens. J Clin Microbiol 45, 906-914.

Siemann, M. \& Koch-Dorfler, M. (2001). The Platelia Aspergillus ELISA in diagnosis of invasive pulmonary aspergillosis (IPA). Mycoses 44, 266-272.

Skladny, H., Buchheidt, D., Baust, C., Krieg-Schneider, F., Seifarth, W., Leib-Mosch, C. \& Hehlmann, R. (1999). Specific detection of Aspergillus species in blood and bronchoalveolar lavage samples of immunocompromised patients by two-step PCR. J Clin Microbiol 37, 3865-3871.

Steinbach, W. J. (2005a). New findings and unique aspects in pediatric aspergillosis. Med Mycol 43 (Suppl. 1), S261-S265.

Steinbach, W. J. (2005b). Pediatric aspergillosis: disease and treatment differences in children. Pediatr Infect Dis J 24, 358-364.

Steinbach, W. J., Addison, R. M., McLaughlin, L., Gerrald, Q., Martin, P. L., Driscoll, T., Bentsen, C., Perfect, J. R. \& Alexander, B. D. (2007). Prospective Aspergillus galactomannan antigen testing in pediatric hematopoietic stem cell transplant recipients. Pediatr Infect Dis J 26, $558-564$.

Subira, M., Martino, R., Rovira, M., Vazquez, L., Serrano, D. \& De La Cámara, R. (2003). Clinical applicability of the new EORTC/MSG classification for invasive pulmonary aspergillosis in patients with hematological malignancies and autopsy-confirmed invasive aspergillosis. Ann Hematol 82, 80-82. 
Sulahian, A., Touratier, S. \& Ribaud, P. (2003). False positive test for aspergillus antigenemia related to concomitant administration of piperacillin and tazobactam. N Engl J Med 349, 2366-2367.

Upton, A. \& Marr, K. A. (2006). Emergence of opportunistic mould infections in the hematopoietic stem cell transplant patient. Curr Infect Dis Rep 8, 434-441.

Viscoli, C., Machetti, M., Cappellano, P., Bucci, B., Bruzzi, P., Van Lint, M. T. \& Bacigalupo, A. (2004). False-positive galactomannan platelia Aspergillus test results for patients receiving piperacillintazobactam. Clin Infect Dis 38, 913-916.

Walsh, T. J., Gonzalez, C., Lyman, C. A., Chanock, S. J. \& Pizzo, P. A. (1996). Invasive fungal infections in children: recent advances in diagnosis and treatment. Adv Pediatr Infect Dis 11, 187-290.

Walsh, T. J., Shoham, S., Petraitiene, R., Sein, T., Schaufele, R., Kelaher, A., Murray, H., Mya-San, C., Bacher, J. \& Petraitis, V. (2004a). Detection of galactomannan antigenemia in patients receiving piperacillin-tazobactam and correlations between in vitro, in vivo, and clinical properties of the drug-antigen interaction. J Clin Microbiol 42, 4744-4748.

Walsh, T. J., Teppler, H., Donowitz, G. R., Maertens, J. A., Baden, L. R., Dmoszynska, A., Cornely, O. A., Bourque, M. R., Lupinacci, R. J. \& other authors (2004b). Caspofungin versus liposomal amphotericin $\mathrm{B}$ for empirical antifungal therapy in patients with persistent fever and neutropenia. N Engl J Med 351, 1391-1402.

White, P. L., Linton, C. J., Perry, M. D., Johnson, E. M. \& Barnes, R. A. (2006). The evolution and evaluation of a whole blood polymerase chain reaction assay for the detection of invasive aspergillosis in hematology patients in a routine clinical setting. Clin Infect Dis 42, 479-486.

Zaoutis, T. E., Heydon, K., Chu, J. H., Walsh, T. J. \& Steinbach, W. J. (2006). Epidemiology, outcomes, and costs of invasive aspergillosis in immunocompromised children in the United States, 2000. Pediatrics 117, e711-e716. 